\title{
Spatiotemporal delivery of nanoformulated liraglutide for cardiac regeneration after myocardial infarction
}

This article was published in the following Dove Press journal:

International Journal of Nanomedicine

7 July 2017

Number of times this article has been viewed

\author{
Quan Qi, ${ }^{1, *}$ Lei Lu, ${ }^{2,3, *}$ \\ Haiqing Li, ,** Zhize Yuan,' \\ Gaoxian Chen, 2,3 Miao \\ Lin, ${ }^{2,3}$ Zhengwen Ruan, ${ }^{4}$ \\ Xiaofeng Ye,' Zeyu Xiao, 2,3,5 \\ Qiang Zhao' \\ 'Department of Cardiac Surgery, \\ Rui Jin Hospital, ${ }^{2}$ Department of \\ Pharmacology, Institute of Medical \\ Sciences, ${ }^{3}$ Translational Medicine \\ Collaborative Innovation Center, \\ Shanghai Jiao Tong University \\ School of Medicine, Shanghai, \\ ${ }^{4}$ Department of Cardiology, Yuyao \\ People's Hospital, Yuyao, Zhejiang, \\ ${ }^{5}$ Collaborative Innovation Center of \\ Systems Biomedicine, Shanghai Jiao \\ Tong University School of Medicine, \\ Shanghai, People's Republic of China \\ *These authors contributed equally \\ to this work
}

\footnotetext{
Correspondence: Qiang Zhao; Xiaofeng Ye

Department of Cardiac Surgery, Rui jin Hospital, Shanghai Jiao Tong University School of Medicine, No 197 Rui Jin Er Road, Shanghai 200025, People's Republic of China

Tel +862134186000

Fax +862164333548

Email zq11607@rih.com.cn;

xiaofengye@hotmail.com
}

Zeyu Xiao

Department of Pharmacology, Institute of Medical Sciences, Shanghai Jiao Tong University School of Medicine, No 280 South Chongqing Road, Shanghai 200025, People's Republic of China

Tel/fax +86 2I 63846590x7764I5

Email zxiao@sjtu.edu.cn

\begin{abstract}
The local, intramyocardial injection of proteins into the infarcted heart is an attractive option to initiate cardiac regeneration after myocardial infarction (MI). Liraglutide, which was developed as a treatment for type 2 diabetes, has been implicated as one of the most promising protein candidates in cardiac regeneration. A significant challenge to the therapeutic use of this protein is its short half-life in vivo. In this study, we evaluated the therapeutic effects and long-term retention of liraglutide loaded in poly(lactic-co-glycolic acid)-poly(ethylene glycol) (PLGA-PEG) nanoparticles (NP-liraglutide) on experimental MI. PLGA-PEG nanoparticles (NPs) have been shown to efficiently load liraglutide and release bioactive liraglutide in a sustained manner. For in vitro test, the released liraglutide retained bioactivity, as measured by its ability to activate liraglutide signaling pathways. Next, we compared the effects of an intramyocardial injection of saline, empty NPs, free liraglutide and NP-liraglutide in a rat model of MI. NPs were detected in the myocardium for up to 4 weeks. More importantly, an intramyocardial injection of NP-liraglutide was sufficient to improve cardiac function $(P<0.05)$, attenuate the infarct size $(P<0.05)$, preserve wall thickness $(P<0.05)$, promote angiogenesis $(P<0.05)$ and prevent cardiomyocyte apoptosis $(P<0.05)$ at 4 weeks after injection without affecting glucose levels. The local, controlled, intramyocardial delivery of NP-liraglutide represents an effective and promising strategy for the treatment of MI.
\end{abstract}

Keywords: spatiotemporal delivery, nanoformulated liraglutide, cardiac regeneration

\section{Introduction}

Heart failure (HF) after myocardial infarction (MI) is the leading cause of death, with an estimated 7.3 million deaths per year worldwide. ${ }^{1}$ Despite their clinical importance, current treatment methods are insufficient for the control of HF after MI. ${ }^{2}$ Some accepted treatment methods for MI still have several limitations. ${ }^{3}$ Thus, several new therapies have been developed to regenerate the infarcted myocardium, such as the administration of stem cells, ${ }^{4}$ growth factors or proteins ${ }^{5}$ or gene therapy. ${ }^{6}$ Liraglutide has initially been approved for clinical use as a treatment for type 2 diabetes. ${ }^{7}$ Recently, liraglutide has been suggested to be beneficial for MI, independent of glycemic control. ${ }^{7,8}$ In fact, the therapeutic benefits of liraglutide have been reported in several studies, including improved cardiac performance, ${ }^{9}$ an attenuated infarct size,${ }^{10}$ increased myocardial blood flow, ${ }^{11}$ inhibition of myocardial apoptosis ${ }^{12}$ and activation of myocardial signaling pathways. ${ }^{13}$ Furthermore, the Liraglutide Effect and Action in Diabetes: Evaluation of Cardiovascular Outcome Results (LEADER) trial, a randomized, double-blind, placebo-controlled clinical trial with long-term follow-up, confirmed the favorable effects of liraglutide on MI. ${ }^{8}$ 
However, like many other proteins, the clinical applicability of liraglutide is limited by its short half-life (13 h) under biological conditions, thereby requiring repeated subcutaneous injections. ${ }^{14}$ Furthermore, subcutaneous administration of liraglutide is associated with undesired systemic side effects, such as a relatively higher frequency of nausea, diarrhea and vomiting. ${ }^{15}$ Hypoglycemia is infrequent but is still observed, particularly when liraglutide is used in combination with other hypoglycemics. ${ }^{14}$ A relatively high percentage of subjects withdrew from the clinical trial due to systemic adverse events $(5 \%){ }^{16}$

In this study, we propose spatiotemporal delivery of liraglutide-loaded poly(ethylene glycol) (PEG)-modified poly(lactic-co-glycolic acid) (PLGA) nanoparticles (NPliraglutide) into the infarcted myocardium as a more appealing and promising therapeutic approach for treating MI. For spatial delivery, NP-liraglutide is locally injected into the myocardium through a mini-thoracotomy injection. Such a locally intramyocardial delivery of liraglutide leads to minimal tissue exposure, thus reducing the undesired systemic side effects. For temporal delivery, NP-liraglutide delivery systems allow the release behavior of liraglutide to be controlled over a prolonged period of 4 weeks, thus overcoming challenges posed by its short half-life. Our team has proven experience in the development of biodegradable PLGA-PEG nanoparticles (NPs). ${ }^{17-20}$ We extend this study to a further exploration of the possibility of NP-liraglutide delivery systems to treat MI. We hypothesized that the controlled delivery of liraglutide from PLGA-PEG NPs would maximize its therapeutic effects on MI.

\section{Materials and methods Preparation of PLGA-PEG NPs containing liraglutide}

An overview of the work flow is demonstrated in Figure 1. Liraglutide was purchased from Creative Peptides (Shirley, NY, USA). PLGA (poly-D,L-lactide/glycolide 50/50; molecular weight $[\mathrm{MW}]=30 \mathrm{kDa}$ ) was purchased from Jinan Daigang Biomaterial Co., Ltd. (Jinan, China). PEG $\left(\mathrm{NH}_{2}-\right.$ PEG-COOH, MW =5 kDa) was obtained from JenKem Technology (Tianjin, China). Poly(vinyl alcohol) (PVA217 , degree of polymerization 1,700, degree of hydrolysis $88.5 \%, \mathrm{MW}=72-84 \mathrm{kDa}$ ) was purchased from Kuraray (Osaka, Japan). Acetonitrile and potassium dihydrogen phosphate (both in high-pressure liquid chromatography [HPLC] grade) were obtained from Tedia (Cincinnati, OH, USA). We synthesized PLGA-PEG using the classical method. ${ }^{21}$ NP-liraglutide were prepared with the double emulsion

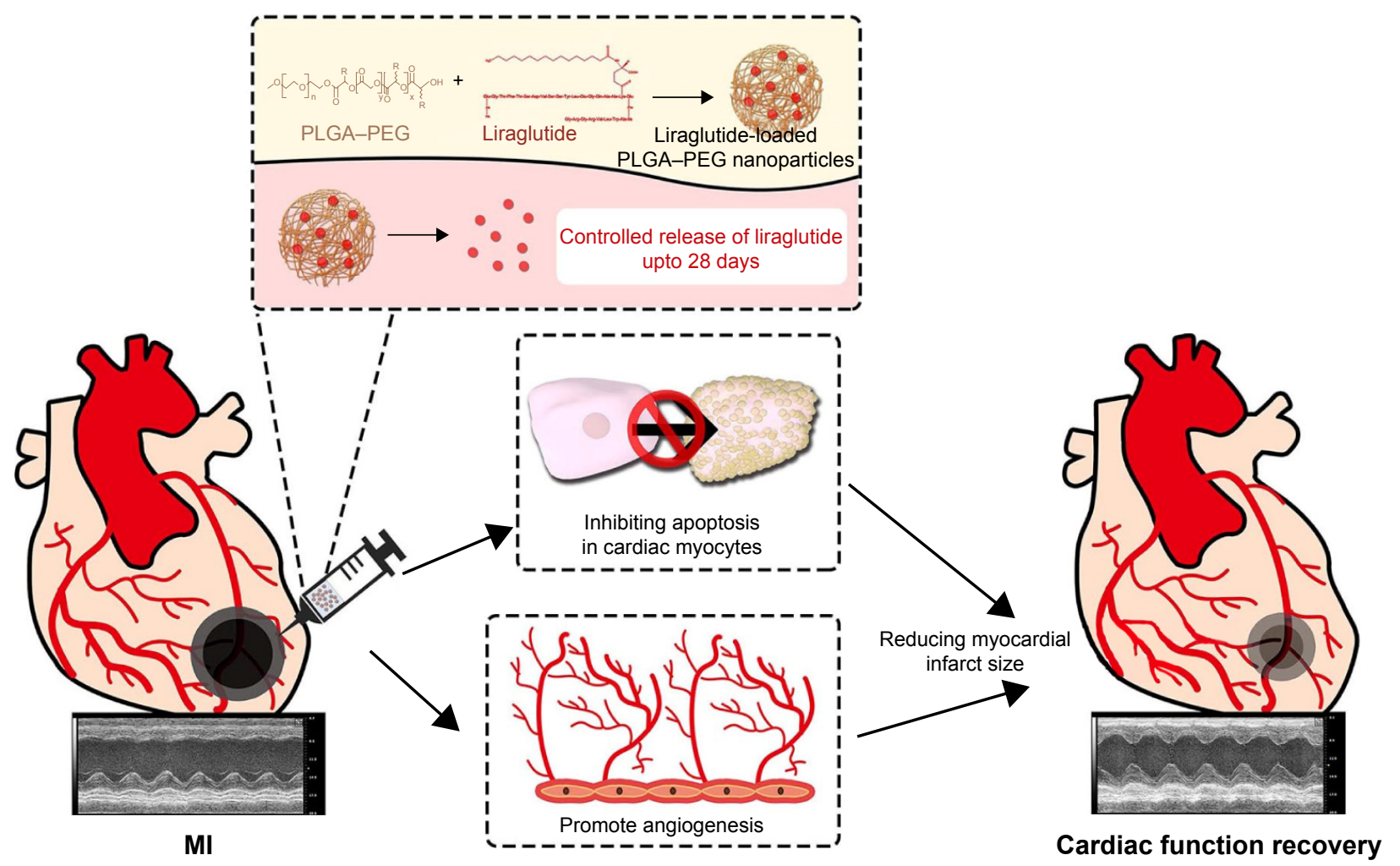

Figure I Schematic diagram of the work flow.

Abbreviations: MI, myocardial infarction; PEG, poly(ethylene glycol); PLGA, poly(lactic-co-glycolic acid). 
method. First, $3.8 \mathrm{mg}$ of liraglutide was dissolved in $0.5 \mathrm{~mL}$ of a $1 \%(\mathrm{w} / \mathrm{v})$ aqueous sodium acetate solution with $2 \%$ PVA, and $25 \mathrm{mg}$ of PLGA-PEG was dissolved in $2.5 \mathrm{~mL}$ of dichloromethane (DCM). ${ }^{22}$ The solution was then emulsified by sonication on ice in $12.5 \mathrm{~mL}$ of a $2 \%$ PVA solution using a probe sonicator. Then, the emulsion was stirred at $550 \mathrm{rpm}$ for $3 \mathrm{~h}$ to evaporate the solvent. The obtained NPs were collected by centrifugation at 4,000 rpm for $25 \mathrm{~min}$ and washed three times with water.

\section{Characterization of the PLGA-PEG NPs and measurement of liraglutide release}

The particle size, size distribution and zeta potential of the NPs were determined by dynamic light scattering (DLS) analysis using a Zetasizer Nano ZS instrument (Malvern, Worcestershire, UK). The morphology of the NPs was determined using transmission electron microscopy (TEM; H-600; Hitachi, Tokyo, Japan) immediately after the samples were negatively stained with sodium phosphotungstate. To determine the drug encapsulation efficiency (EE) of the liraglutide-loaded NPs, a predetermined amount of NPs was dissolved in acetonitrile to release the liraglutide. The resulting mixture was agitated to ensure complete dissolution, then filtered and analyzed by reverse-phase HPLC (L-2000; Hitachi) with a $\mathrm{C} 18$ column (dimensions of $250 \times 4.6 \mathrm{~mm}$ ) to determine the liraglutide concentrations. The mobile phase consisted of acetonitrile (solvent $\mathrm{A}$ ) and a 0.05-M aqueous $\mathrm{KH}_{2} \mathrm{PO}_{4}$ solution ( $\mathrm{pH} 4.0$, solvent $\mathrm{B}$ ). The flow rate was set to $1.0 \mathrm{~mL} / \mathrm{min}$. NP samples $(100 \mu \mathrm{L})$ at $0.02 \mathrm{mg} / \mathrm{mL}$, enclosed in a mini dialysis unit (Thermo Fisher Scientific, Waltham, MA, USA), were incubated in $1 \mathrm{~mL}$ PBS ( $\mathrm{pH} \mathrm{7.4)}$ ) at $37^{\circ} \mathrm{C}$ under 100-rpm orbital shaking. Thereafter, $100 \mu \mathrm{L}$ samples were taken from the incubation medium and analyzed for liraglutide by HPLC as described earlier. After sampling, the incubation medium was replenished with blank PBS. The controlled release of liraglutide from the NPs was measured for 4 weeks.

\section{In vitro bioactivity of the released liraglutide}

The bioactivity of the released liraglutide was assessed by Western blotting to measure the phosphorylation level of AKT protein kinase in vitro. The procedure used to isolate the cells in this study was approved by the Institutional Review Committee of Shanghai Jiao Tong University School of Medicine. Neonatal ventricular myocytes were obtained from 0- to 3-day-old Sprague Dawley (SD) rats as previously described. ${ }^{23}$ The collected cells were pre-plated for
$30 \mathrm{~min}$ at $37^{\circ} \mathrm{C}$. Then, the cardiomyocyte-enriched fraction was plated in six-well plates $\left(1 \times 10^{6}\right.$ cells/well $)$. The neonatal ventricular myocytes were cultured in cardiac myocyte medium (CMM; ScienCell, Carlsbad, CA, USA), and the culture medium was changed every 2 days. After serum starvation for $24 \mathrm{~h}$, the 7-day-old neonatal cardiomyocytes were washed with fresh medium without serum and treated with the conditioned media from the liraglutide release system in the first 3 days, which has been diluted to $100 \mathrm{nmol} / \mathrm{L}$ liraglutide, for $20 \mathrm{~min} ;{ }^{22}$ cells cultured with plain medium, free liraglutide (100 nmol/L) or non-loaded NPs were used as the control groups. After the incubation, the medium was removed and the wells containing cells were quickly frozen in liquid nitrogen and stored at $-80^{\circ} \mathrm{C}$. Western blot analyses were performed using a previously described method. ${ }^{24}$ After blocking, the membranes were incubated with anti-pAKT and anti-total AKT antibodies (1:1,000 dilution; Cell Signaling Technology, Beverly, MA, USA), incubated with corresponding horseradish peroxidase (HRP)-conjugated anti-rabbit secondary antibodies (1:5,000 dilution; Hua An, Hangzhou, China) and developed using the standard enhanced chemiluminescence (ECL) procedure (Pierce, Rockford, IL, USA). Blots were visualized using Biomax film (Kodak, Rochester, NY, USA). Densitometric analyses were performed using the Image-pro plus6.0 (Media Cybernetics, Silver Spring, MD, USA).

\section{In vivo studies of the rat $\mathrm{MI}$ model}

SD rats were obtained from the Laboratory Animal Care Facility of Shanghai Jiao Tong University School of Medicine. All the procedures in this study conformed to the "Guide for the Care and Use of Laboratory Animals" (National Institutes of Health, Publication No 85-23, Revised) and were performed under the supervision of the Shanghai Jiao Tong University Institutional Animal Care and Use Committee. Male SD rats (200-250 g) were anesthetized with pentobarbital sodium $(30 \mathrm{mg} / \mathrm{kg}$, intraperitoneal), intubated and subjected to left anterior descending coronary artery (LAD) ligation below the atrium (Video S1). The result was confirmed by an increase in the electrocardiogram ST-segment and regional cyanosis. The baseline echocardiographic measurements were collected 2 days following MI. ${ }^{25}$ After the baseline and screening echocardiograms, 40 rats were randomly divided into four groups. Next, the chests were reopened, and $100 \mu \mathrm{L}$ of NP-liraglutide $(n=8$; $0.38 \mathrm{mg}$ of liraglutide), the same amount of liraglutide-loaded saline (liraglutide, $\mathrm{n}=8 ; 0.38 \mathrm{mg}$ of liraglutide), non-loaded PLGA-PEG NPs $(n=7)$ or saline solution $(n=9)$ was injected 
into four points around the infarct areas at a depth of $\approx 3 \mathrm{~mm}$ with a $29-\mathrm{G}$ insulin syringe $(25 \mu \mathrm{L} /$ injection $)$ (Video $\mathrm{S} 2)$. For the sham group $(n=8)$, a suture was placed around the LAD but was not ligated.

\section{Confirmation of the in vivo visualization of the NPs in the myocardium}

A group of 16 infarcted rats was euthanized 1, 3, 5, 7, 14, 21 and 28 days after the injection of the fluorescent-labeled NPs (drug-free; $n=2$ ). Rhodamine B was applied as a fluorescent marker for in vivo follow-up. The same amount of rhodamine-labeled NPs was injected into the peri-infarcted areas. The sections were stained with 4-6-diamidino2-phenylindole (DAPI; $5 \mu \mathrm{L} /$ section; Sigma-Aldrich, St Louis, MO, USA) to label the nucleus.

\section{Echocardiography for evaluating cardiac function}

The left ventricular(LV) performance and structural remodeling were assessed by echocardiography using the Vevo 2100 Imaging System (VisualSonics, Toronto, ON, Canada). Echocardiographic data were collected and analyzed 2 days after ligation (baseline) and 4 weeks after injection. ${ }^{26}$ The LV internal systolic dimension (LVIDs) and LV internal diastolic dimension (LVIDd) were measured by tracing the endocardial border to calculate the LV ejection fraction (EF) and fractional shortening (FS) using the following equations: $\mathrm{EF}\left(\mathrm{LVIDd}^{3}-\mathrm{LVIDs}^{3}\right) / \mathrm{LVIDd}^{3} \times 100 \%$ and FS (LVIDd LVIDs)/LVIDd $\times 100 \%$. All measurements were performed by two independent, qualified echo operators.

\section{Histological analysis of the LV cavity areas and scar areas}

The rats were euthanized, and the hearts were collected 4 weeks following the injection. The hearts were perfused with 4\% formaldehyde for $20 \mathrm{~min}$, rinsed with PBS for approximately $10 \mathrm{~min}$ and sectioned into five slices in the transverse orientation below the ligature to the apex at 2-mm intervals. The heart samples were fixed, paraffin-embedded and sectioned into 5- $\mu \mathrm{m}$ slices. The histological analysis was performed on Masson's trichrome-stained slides from the closest section plane ${ }^{27}$ The following data were measured using Image J software: ventricular wall thickness, septum thickness $(\mathrm{mm})$, scar thickness $(\mathrm{mm}), \mathrm{LV}$ cavity area $\left(\mathrm{mm}^{2}\right)$ and whole $\mathrm{LV}$ area $\left(\mathrm{mm}^{2}\right)$. Each measurement was performed three times. Then, the average of each parameter was calculated. Relative scar thickness was calculated as the ratio of the average thickness of the scar wall to the average thickness of the uninfarcted wall. The infarct expansion index was defined as follows: (LV cavity area/whole LV area)/relative scar thickness. ${ }^{23}$

\section{Immunohistochemical measurements of neovascularization}

An immunofluorescence analysis was carried out to identify blood vessel formation. Blood vessel staining was performed with a mouse monoclonal antibody against CD31 (1:100; Abcam, Cambridge, MA, USA) and a rabbit polyclonal antibody against alpha smooth muscle actin ( $\alpha$-SMA; 1:200; Abcam), with the following secondary antibodies: anti-rabbit Alexa Fluor $^{\circledR} 594$ (A21207; 1/1,000; Thermo Fisher Scientific) and biotin-streptavidin Alexa Fluor ${ }^{\circledR} 488$ (BA-2001, 1:200; Thermo Fisher Scientific - 532354, 1:150, Thermo Fisher Scientific). For confocal imaging, an LSM710 Meta confocal microscope (Carl Zeiss, Feldbach, Switzerland) was used. $\alpha$-SMA and CD31-positive structures were identified and photographed. Arterioles and capillaries bordering the infarct at the mid-infarct level were identified by counting the $\alpha$-SMA- and CD31-positive structures, subsequently computed using a fluorescence microscope in a blinded manner from eight randomly selected images as the mean within $1-\mathrm{mm}^{2}$ areas. The maturation index was calculated based on the number of $\alpha$-SMA-positive vessels relative to the total number of vessels. ${ }^{28}$ All data analyses were performed in a blinded manner. All measurements were performed using Image $\mathbf{J}$ software.

\section{TUNEL (terminal deoxynucleotidyl transferase dUTP nick-end labeling) staining to measure apoptosis}

TUNEL staining was performed according to the manufacturer's instructions. The nicked ends of DNA were visualized with Fluorescence (number 11684795910; Hoffman-La Roche Ltd., Basel, Switzerland). Apoptotic nuclei and total nuclei bordering the infarct at the mid-infarct level were identified and subsequently computed using a fluorescence microscope in a blinded manner from three randomly selected fields $(200 \times$ magnification). The numbers of TUNEL-positive nuclei or total nuclei were calculated using Image $J$ software. The percentage of TUNEL-positive nuclei was defined as the number of apoptotic nuclei compared to the total number of nuclei. ${ }^{29}$

\section{Blood glucose levels}

Blood samples of rats from four groups were collected. Nonfasting blood glucose was randomly measured at 1, 2, 3 and 4 weeks after injection by an Automatic Biochemical Analyzer (Hitachi). 


\section{Statistical analysis}

All variables are presented as mean \pm standard error of the mean (SEM) and were calculated using SPSS19.0 (IBM) statistics software. Comparisons of the densitometry, echocardiography data, morphology, blood vessel analysis, apoptotic cell counts and blood glucose levels among groups were performed using one-way ANOVA with Tukey's post hoc test. $P<0.05$ was considered statistically significant.

\section{Results}

\section{Characterization and in vitro delivery of the NP-liraglutide}

In this study, the double emulsion method was used to synthesize spherical PLGA-PEG NPs loaded with liraglutide. TEM observation showed a rough structure of the
NPs (Figure 2A). The size of the PLGA-PEG NPs was $350 \pm 1.83 \mathrm{~nm}$ (Figure 2B), with a zeta potential of $-0.369 \mathrm{mV}$. The EE of liraglutide was $95 \%$ for the PLGA-PEG NPs, which corresponded to a final loading of $95 \mu \mathrm{g}$ of liraglutide per milligram of NPs. In summary, although the PEG coating neutralized the zeta potential of the NPs and slightly increased the particle size, the NPs maintained their negative zeta potential and shape. Importantly, the EE remained at a high level. The controlled release of liraglutide from the NPs was achieved over 4 weeks (Figure 2C). The large variation in the release behavior at the early stage is likely due to the burst release of liraglutide from PLGA-PEG. Approximately 50\% cumulative release was observed on the 7 th day; thereafter, a sustained, very slow rate of liraglutide release was observed. On the 28 th day, approximately $100 \%$ of the liraglutide was
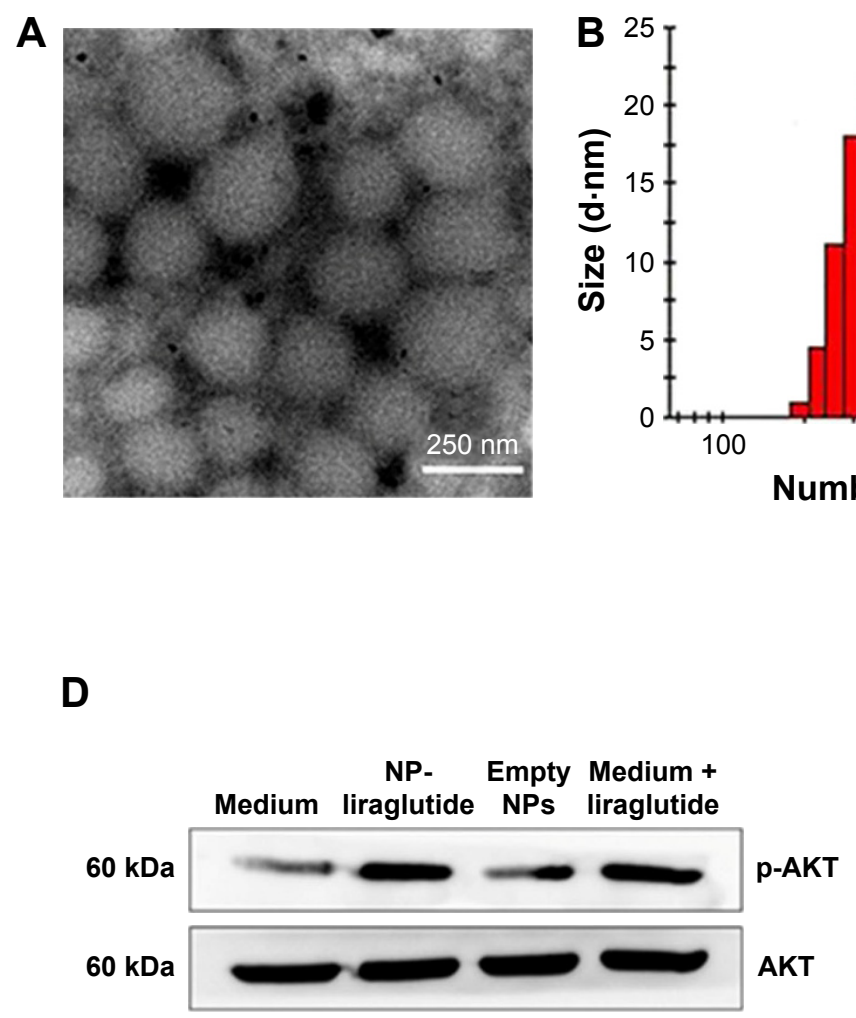
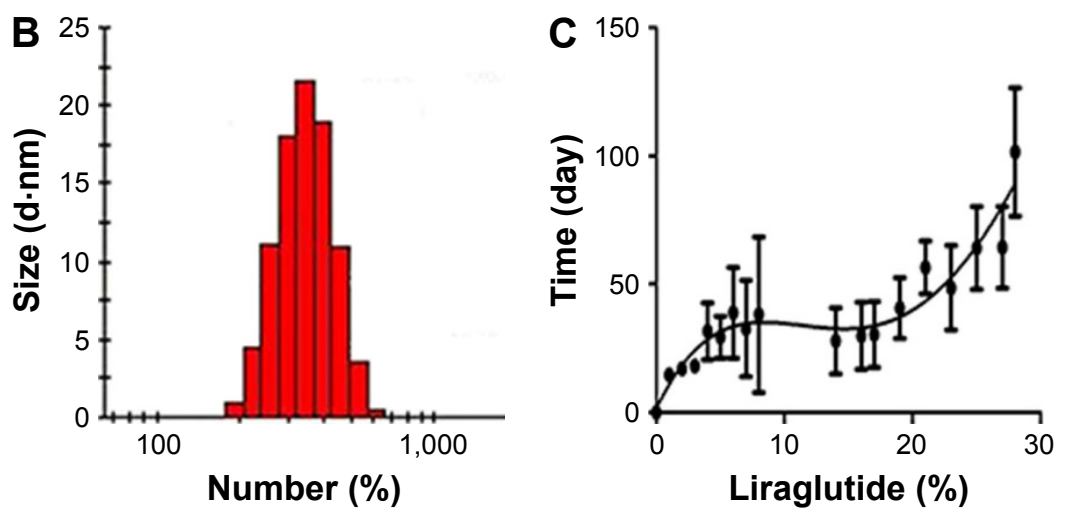

E

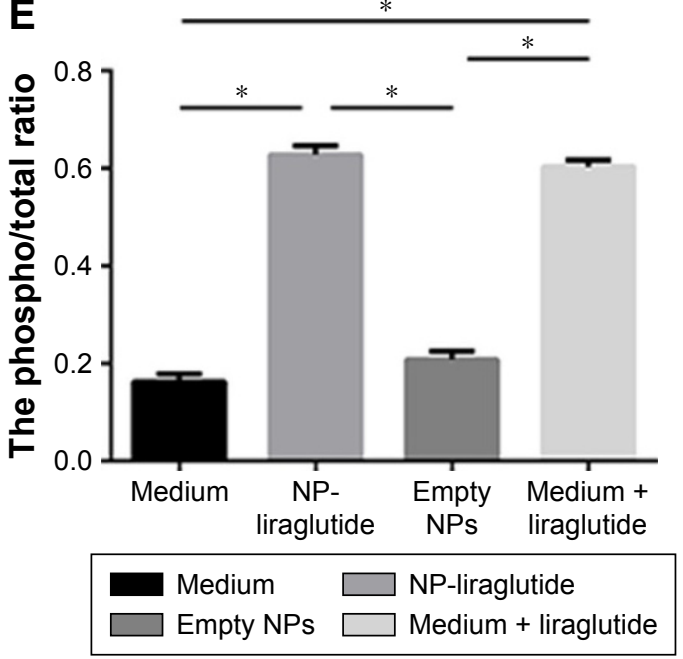

Figure 2 Characterization of NP-liraglutide.

Notes: (A) Transmission electron microscopic images of the NP-liraglutide. Scale bar, $250 \mathrm{~nm}$. (B) The size and zeta potential distribution of NP-liraglutide measured by DLS. (C) In vitro cumulative release profiles of liraglutide from NP-liraglutide $(n=3)$. The percentage of cumulative released liraglutide was calculated as follows: (cumulative released liraglutide at a given time point/total amount of liraglutide) $\times 100 \%$. Data are shown as mean \pm SEM of three samples per time points. (D) Induction of AKT phosphorylation in neonatal rat cardiomyocyte cultures by liraglutide released from the NP-liraglutide. Representative Western blots of cardiomyocyte cultures treated with the only medium, released medium from the NP-liraglutide, medium from empty NPs or medium with direct addition of liraglutide. (E) Densitometric analysis for phosphorylation level of AKT protein kinase based on Western blot. Columns in graph were expressed as the ratio of phosphorylated to total protein signal intensity from three independent experiments. Data are shown as mean $\pm \mathrm{SEM}, * \mathrm{P}<0.05$. NP-liraglutide: liraglutide loaded in PLGA-PEG NPs.

Abbreviations: DLS, dynamic light scattering; NP, nanoparticle; PEG, poly(ethylene glycol); PLGA, poly(lactic-co-glycolic acid); SEM, standard error of the mean. 
released, thereby achieving sustained release. In summary, the PLGA-PEG NPs were useful in controlling the release behavior of liraglutide for prolonged periods.

\section{The in vitro bioactivity of the NP-liraglutide}

Within the organism, liraglutide activates AKT protein kinase, a known downstream target of the liraglutide signaling pathway, to initiate cellular signals. ${ }^{10}$ Therefore, the bioactivity of the released liraglutide was measured by Western blotting to determine the level of AKT protein kinase phosphorylation. As shown in Figure 2D and E, the released liraglutide induced AKT phosphorylation compared to plain medium or medium containing empty NPs $(P<0.05)$. No differences were observed between the released liraglutide group and the free liraglutide group, suggesting that the released liraglutide retained bioactivity.

\section{In vivo therapeutic efficacy in the rat model of $\mathrm{Ml}$}

The fate of the NPs was assessed to confirm the extent to which the NPs were retained in the myocardium over prolonged periods (4 weeks). The level of the NPs decreased over time. However, 4 weeks after treatment, some NPs still resided in the myocardium and the rhodamine B marker was observed up to 4 weeks post injection, confirming the suitability of using NPs for the controlled delivery of liraglutide in the heart (Figure 3).
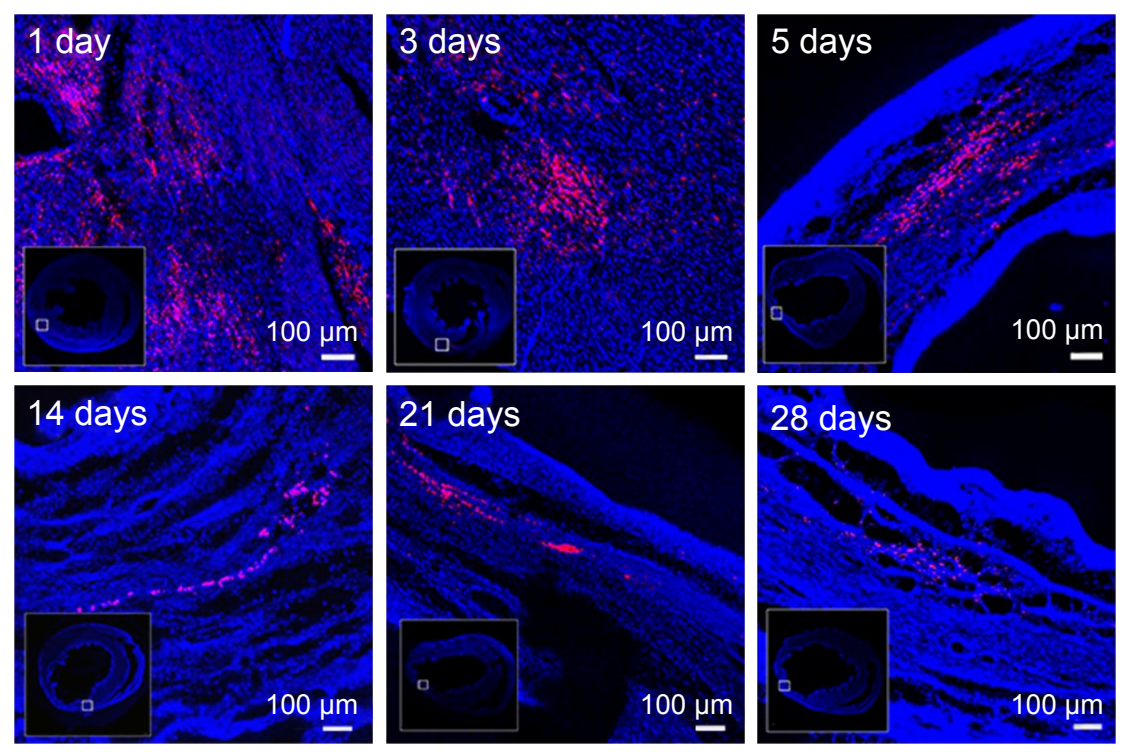

Rats underwent echocardiography 2 days (baseline, 2 days after ligation) and 4 weeks post injection to evaluate cardiac function. Differences in the LVEF, LVFS, LVIDs and LVIDd were not observed among the groups at baseline, indicating a comparable degree of initial cardiac injury (Figure S1). The NP-liraglutide group showed markedly improved LVEF $(56.1 \% \pm 1.3 \%)$ compared to the administration of saline, NP and liraglutide groups $(44.6 \% \pm 3.0 \%, 44.4 \% \pm 2.4 \%$ and $47.6 \% \pm 2.7 \%$, respectively; $P<0.05$ ). Similarly, the injection of NP-liraglutide group significantly increased the LVFS $(30.5 \% \pm 0.8 \%)$ compared to the administration of saline, NP and liraglutide groups $(23.6 \% \pm 1.9 \%, 23.1 \% \pm 1.4 \%$ and $25.1 \% \pm 1.7 \%$, respectively, $P<0.05$ ). Moreover, the NPliraglutide group showed the smallest increase in LVIDs (5.29 \pm 0.21$)$ compared to the saline, NP and liraglutide groups $(6.62 \pm 0.32,6.49 \pm 0.23$ and $6.62 \pm 0.33 \mathrm{~mm}$, respectively, $P<0.05)$. A similar trend was also noted for the LVIDd, where the NP-liraglutide group showed the smallest increases in the LVIDd (7.60 \pm 0.22$)$ compared to the saline, NP and liraglutide groups $(8.60 \pm 0.25,8.44 \pm 0.20$, and $8.82 \pm 0.29 \mathrm{~mm}$, respectively, $P<0.05$; Figure 4$)$.

H\&E staining and Masson's trichrome staining were performed for the histological analysis of the heart samples from each group (Figure 5). The NP-liraglutide treatment significantly increased the relative scar thickness $(0.42 \pm 0.01)$ compared to the saline, NP and liraglutide groups $(0.35 \pm 0.02$, $0.33 \pm 0.03$ and $0.36 \pm 0.02$, respectively, $P<0.05)$. A significantly smaller infarct expansion index was also observed

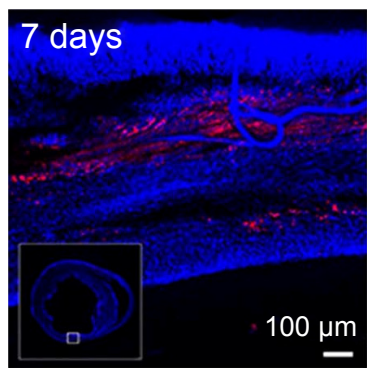

Figure 3 NPs maintaining in the myocardium.

Notes: Representative images show the merging of fluorescent (showing rhodamine NPs in red) and blue field images (showing cell nuclei in blue) at I-, 3-, 5-, 7-, I4-, 2I- and 28-day time points. Scale bars, $100 \mu \mathrm{m}$. Inset pictures are cross sections of representative images.

Abbreviation: NPs, nanoparticles. 
A

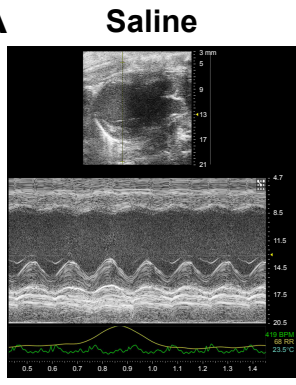

NP

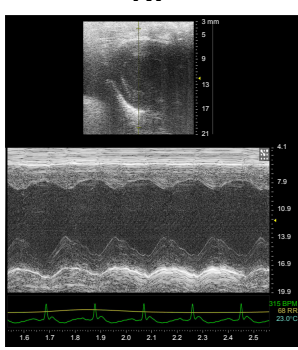

Liraglutide

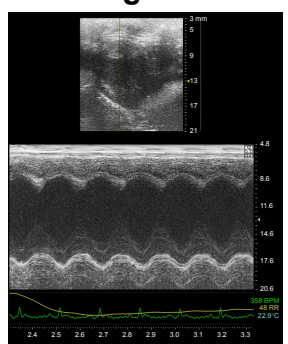

NP-liraglutide

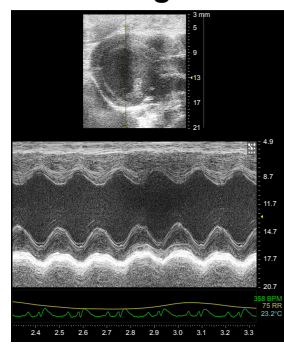

Sham

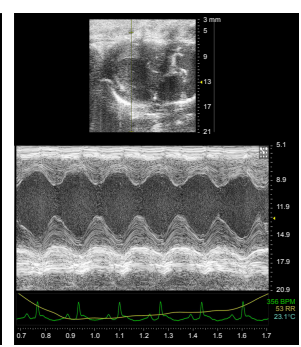

B

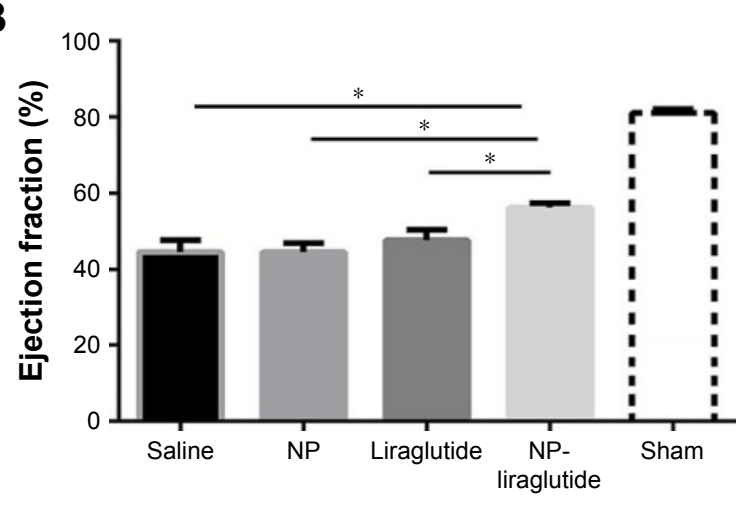

D

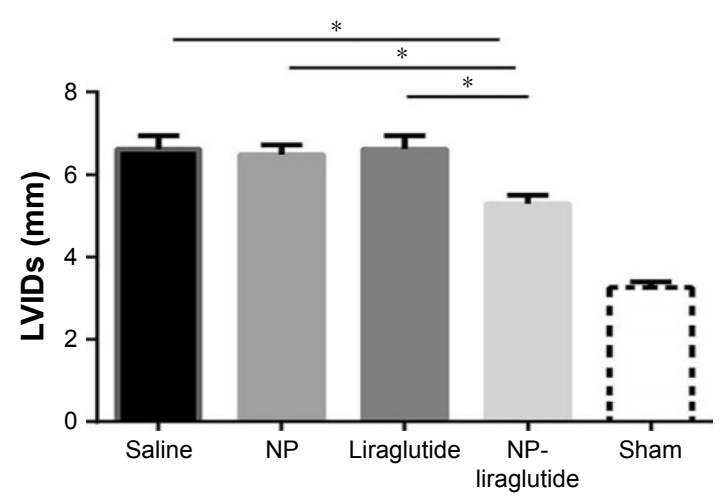

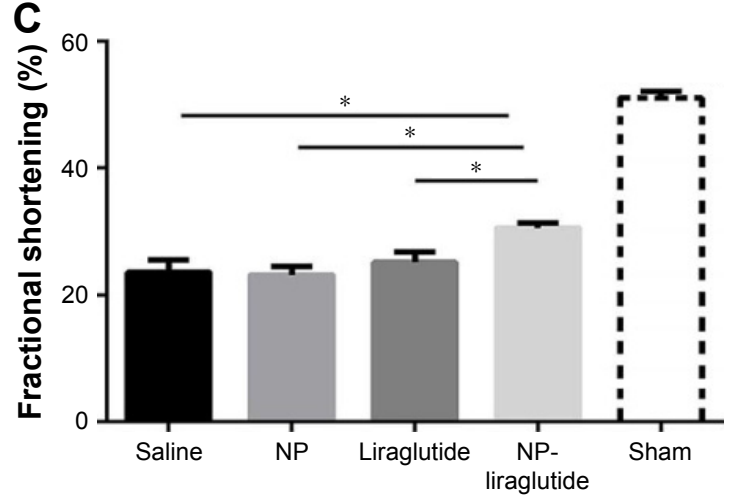

E

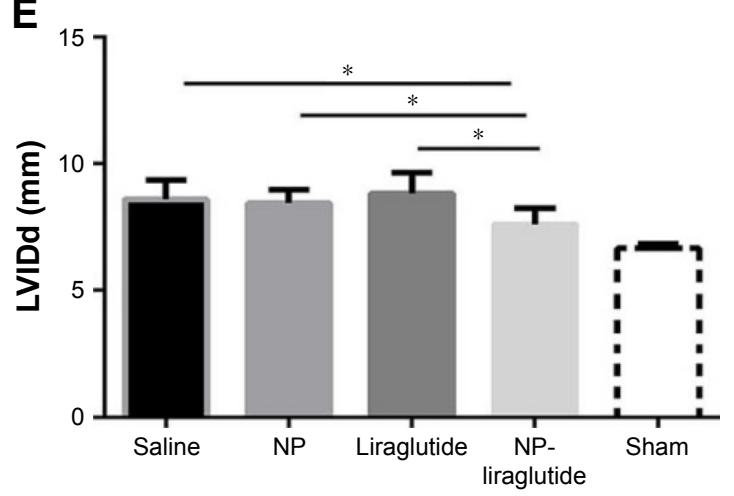

Saline

Liraglutide

NP-liraglutide

Figure 4 Echocardiographic assessments.

Notes: (A) Representative echocardiographic images from each group 4 weeks after treatment. The LVEF (B), LVFS (C), LVIDs (D) and LVIDd (E) were assessed via twodimensional echocardiography. Data are shown as mean \pm SEM, $* P<0.05$. NP-liraglutide: liraglutide loaded in PLGA-PEG NPs.

Abbreviations: LVEF, left ventricular ejection fraction; LVFS, left ventricular fractional shortening; LVIDd, left ventricular internal diastolic dimension; LVIDs, left ventricular internal systolic dimension; NP, nanoparticle; PEG, poly(ethylene glycol); PLGA, poly(lactic-co-glycolic acid); SEM, standard error of the mean.

in the NP-liraglutide group $(0.91 \pm 0.03)$ compared to the saline, NP and liraglutide groups $(1.33 \pm 0.12,1.34 \pm 0.15$ and $1.28 \pm 0.14$, respectively, $P<0.05)$. The MI-induced decreased scar thickness and cardiac dilation (adverse cardiac remodeling) was significantly attenuated by NP-liraglutide. This finding was further confirmed by the results of the angiogenesis and apoptosis assays.

We examined whether the delivery of liraglutide would promote neovascularization using immunohistochemical analyses (Figure 6). The NP-liraglutide treatment exerted superior effects on neovascularization compared to the other treatments. NP-liraglutide treatment induced a greater capillary density in the peri-infarct region $\left(60.18 \pm 1.96 \mathrm{~mm}^{-2}\right)$ compared to the saline, NP and liraglutide groups $(35.91 \pm 3.48$, $39.84 \pm 4.49$ and $41.73 \pm 4.76 \mathrm{~mm}^{-2}$, respectively, $P<0.05$; Figure 6B). Furthermore, as shown in Figure 6C, we found co-localization of CD3 1 and $\alpha$-SMA, indicating that mature vessels were formed. The maturation index in the NP-liraglutide group $(65.5 \% \pm 1.5 \%)$ was increased compared to the saline, NP and liraglutide groups $(26.8 \% \pm 3.4 \%, 27.9 \% \pm 4.3 \%$ and $34.8 \% \pm 3.4 \%$, respectively, $P<0.05$ ), suggesting that NP-liraglutide induced vessel maturation for myocardial 


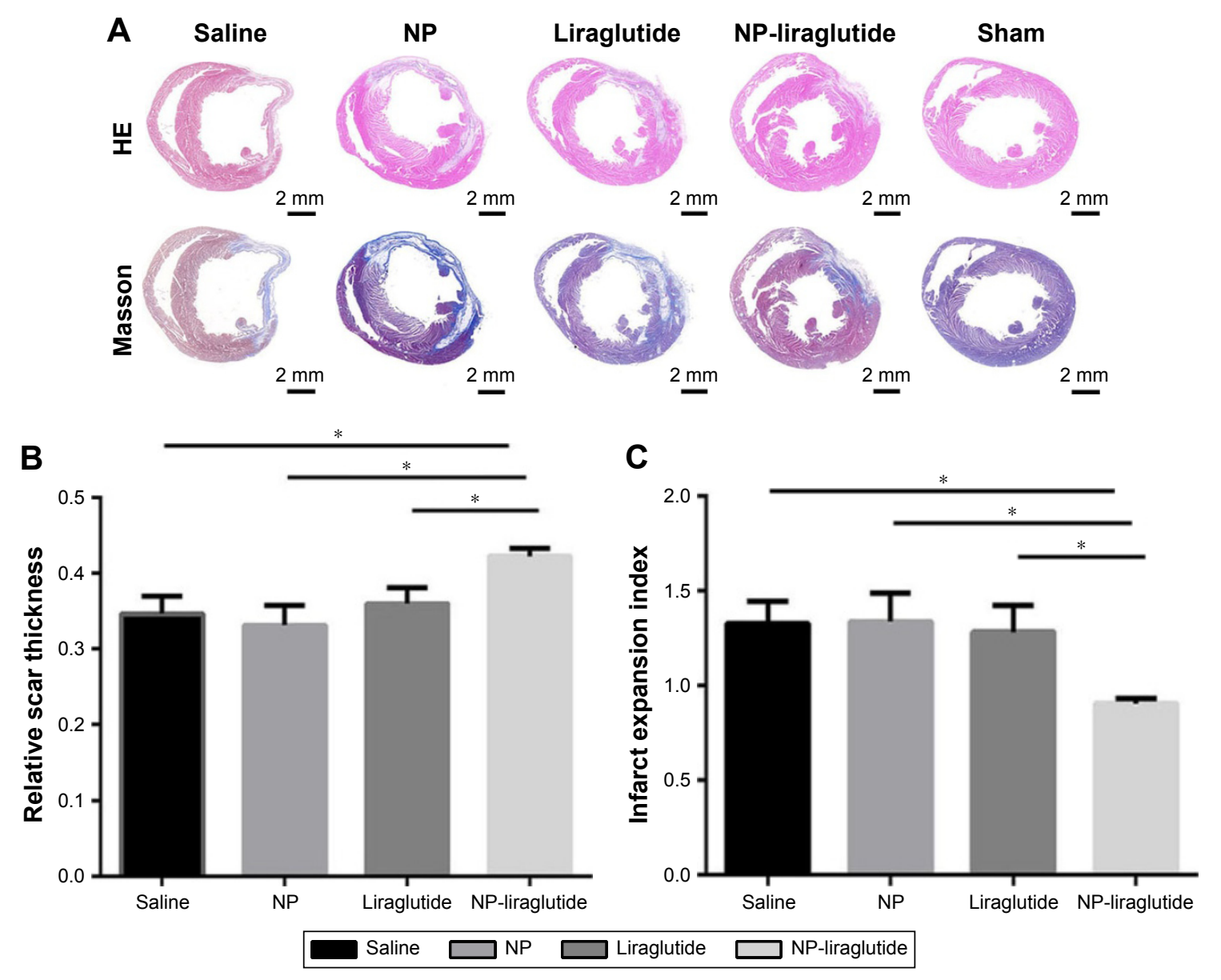

Figure 5 Morphological analysis.

Notes: Morphological analysis of the left ventricle among the different groups. (A) Representative H\&E staining and Masson's trichrome staining. Scale bars, 2 mm. Quantification of the relative scar thickness $(\mathbf{B})$ and infarct expansion index $(\mathbf{C})$ based on Masson's trichrome staining. Data are shown as mean $\pm \mathrm{SEM}$, $* \mathrm{P}<0.05$. NPliraglutide: liraglutide loaded in PLGA-PEG NPs.

Abbreviations: NP, nanoparticle; PEG, poly(ethylene glycol); PLGA, poly(lactic-co-glycolic acid); SEM, standard error of the mean.

regeneration. These data clearly show that the mechanisms underlying the capacity of NP-liraglutide to improve cardiac function and attenuate cardiac remodeling could be related to enhanced angiogenesis.

Cardiomyocyte apoptosis is another leading cause of HF. ${ }^{30}$ A TUNEL assay (Hoffman-La Roche Ltd) was performed to detect the apoptotic cardiac cells in the peri-infarct region 4 weeks after the injection. As shown in Figure 7, the NP-liraglutide group showed a significant reduction in the number of apoptotic cells $(3.0 \% \pm 0.3 \%)$ compared to the saline, NP and liraglutide groups $(5.9 \% \pm 0.7 \%, 5.1 \% \pm 0.8 \%$ and $5.4 \% \pm 0.8 \%$, respectively, $P<0.05$ ). Thus, cardiomyocyte apoptosis is suppressed by the NP-liraglutide treatment.

Liraglutide has been approved for the treatment of diabetes. Therefore, we examined whether the delivery of liraglutide in the heart induced hypoglycemia. Significant differences were not detected among the four groups (Figure S2). Therefore, the cardioprotective effects of NP-liraglutide are attainable without affecting the normal blood glucose levels, consistent with the findings of a previous study. ${ }^{10}$

\section{Discussion}

In this study, we confirmed the suitability of using NPliraglutide for the local, controlled, intramyocardial delivery of liraglutide. In addition, NP-liraglutide may improve cardiac function after MI by relieving adverse cardiac remodeling, inducing angiogenesis and inhibiting cardiac cell apoptosis.

Adverse cardiac remodeling after MI is a compensatory response that causes an irreversible loss of cardiomyocytes and eventually HF. However, the current therapies are mainly palliative. ${ }^{31}$ Thus, new therapies must be developed to overcome the poor prognosis of MI. Several alternative strategies based on drug treatments, ${ }^{32}$ injections of stem cells or proteins, ${ }^{4,5}$ surgical interventions ${ }^{33}$ and epicardial restraint devices $^{34}$ have been used to regenerate the infarcted heart. 
A
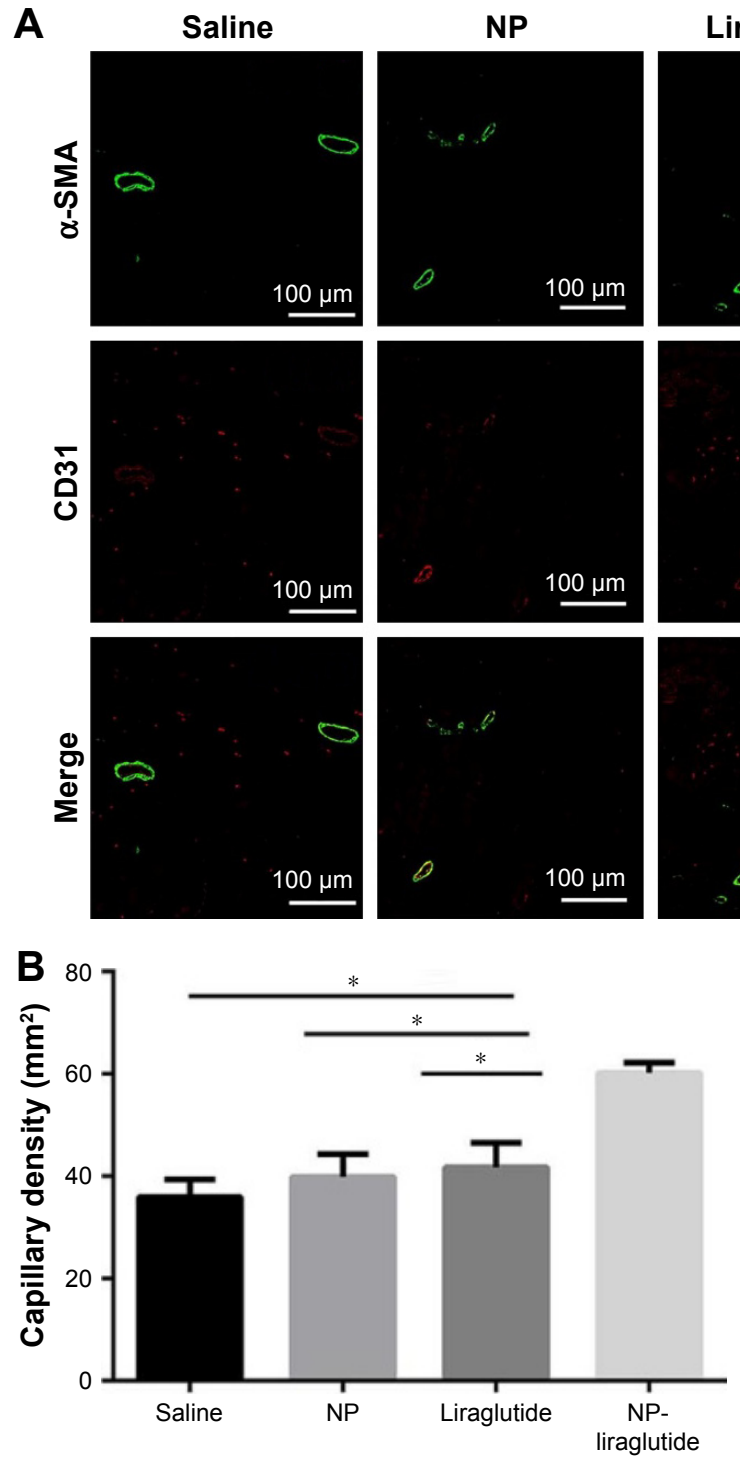

Saline Liraglutide
NP
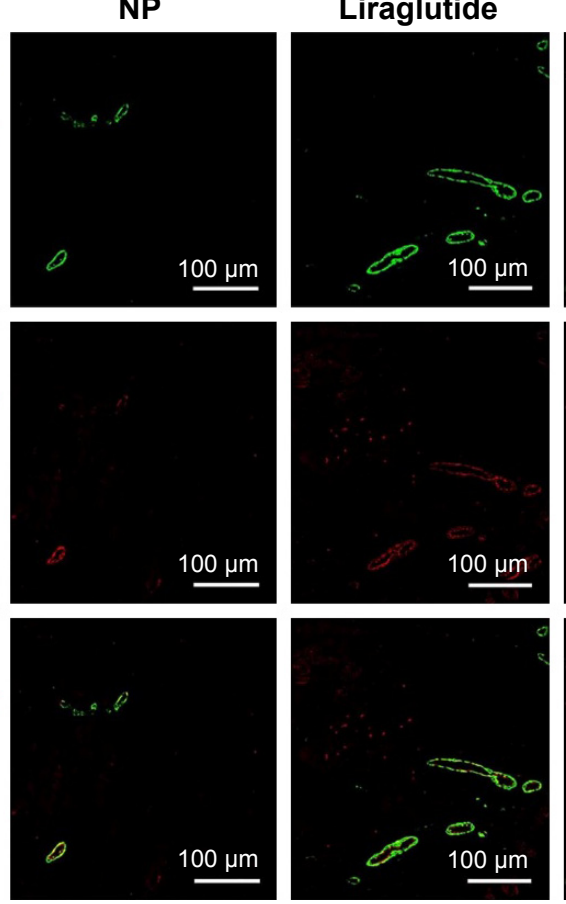

NP-liraglutide
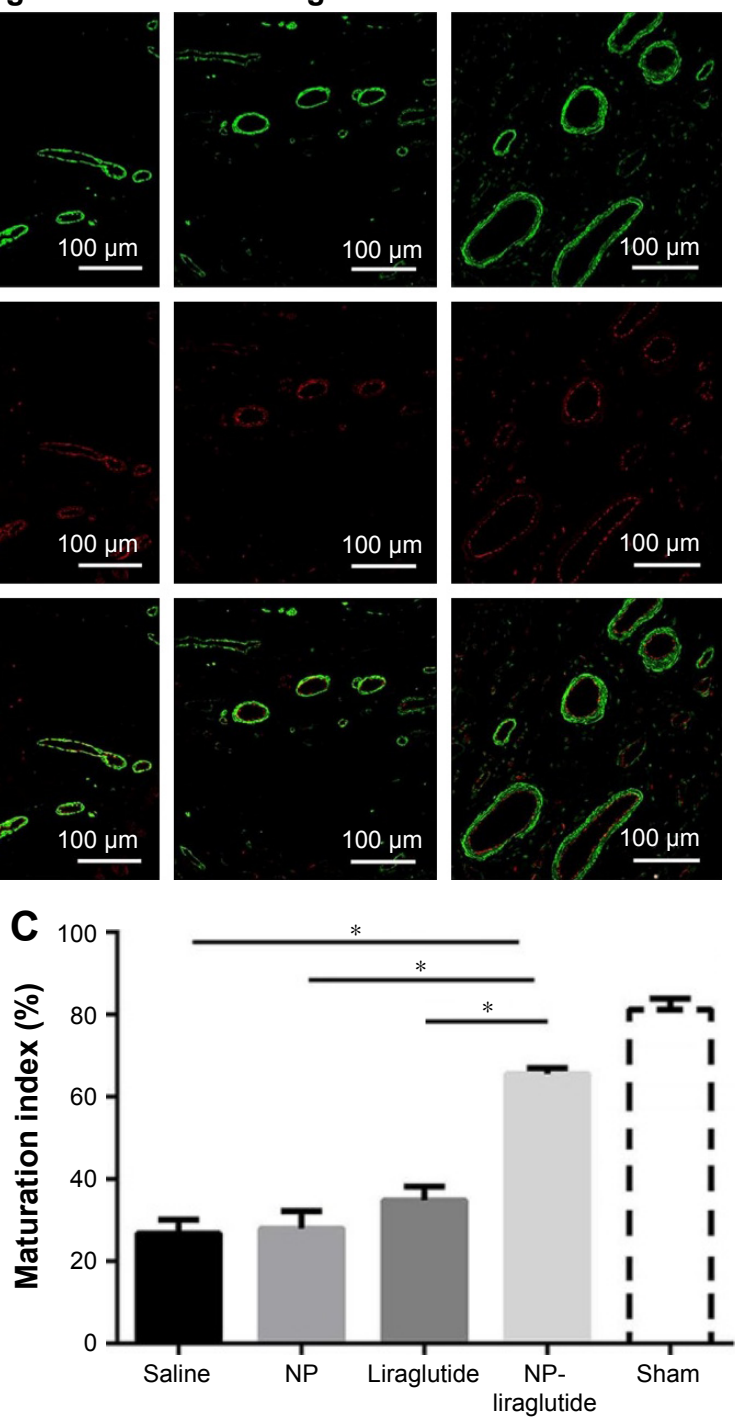

Saline $\square$ NP $\square$ Liraglutide NP-liraglutide J-Z Sham

Figure 6 Immunofluorescence assessment of the vascularization 4 weeks after treatment.

Notes: (A) Representative immunohistochemical staining of blood vessel SMA and ECs in the peri-infarct myocardial tissue. CD3I (red) and $\alpha$-SMA (green) staining for the blood vessels. Scale bars, $100 \mu \mathrm{m}$. Quantification of the capillary density (B) and maturation index (C). Increased capillary density and maturation index are shown in the group treated with the NP-liraglutide relative to the changes in the other groups. Data are shown as mean $\pm S E M$, $* P<0.05$. NP-liraglutide: liraglutide loaded in PLGA-PEG NPs.

Abbreviations: ECs, endothelial cells; NP, nanoparticle; PEG, poly(ethylene glycol); PLGA, poly(lactic-co-glycolic acid); SEM, standard error of the mean; SMA, smooth muscle actin.

The effects of liraglutide have been shown to be beneficial for the treatment of MI. ${ }^{10,35}$ Several factors may be responsible for the cardioprotective effects of liraglutide. First, glucose metabolism is promoted over fatty acid metabolism, which is a more efficient method for oxygen production ${ }^{36}$ and may decrease oxygen demand. Second, liraglutide enhances vascularization. ${ }^{37}$ Finally, liraglutide reduces myocardial apoptosis. ${ }^{10}$

Protein therapy is suggested to be a promising treatment strategy for MI. ${ }^{38}$ However, the short half-lives of proteins in their free form under biological conditions limit their successful application. ${ }^{39}$ Similarly, the clinical application of liraglutide is limited by its short half-life. ${ }^{40}$

PLGA demonstrated great potential by maintaining the local concentrations of proteins for a prolonged period without the necessity for multiple injections. ${ }^{41}$ PLGA is a potential vehicle candidate for the sustained release of proteins that show promise as treatments for cardiac diseases. ${ }^{42-45}$ Moreover, PEG is effective in preventing NP phagocytosis by 

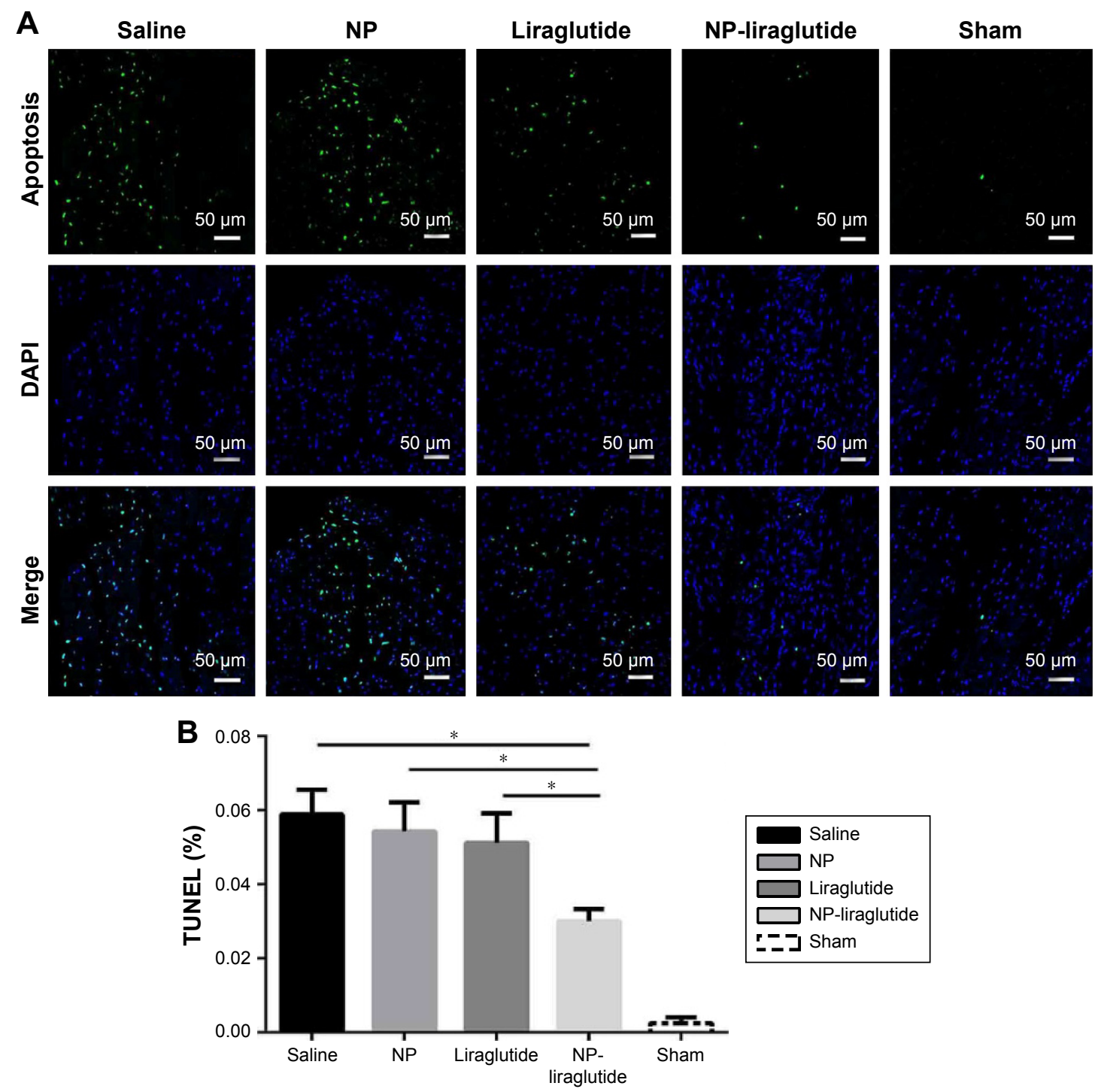

Figure 7 The liraglutide reduces cardiac cell apoptosis after MI.

Notes: (A) Representative photomicrographs of TUNEL staining (green) and DAPI (blue) staining for the saline, NP, liraglutide, NP-liraglutide and sham groups in periinfarct region. Scale bars, $50 \mu \mathrm{m}$. (B) Quantification of the percentage of TUNEL-positive nuclei. Data are shown as mean \pm SEM, $* P<0.05$. NP-liraglutide: liraglutide loaded in PLGA-PEG NPs.

Abbreviations: DAPI, 4-6-diamidino-2-phenylindole; MI, myocardial infarction; NP, nanoparticle; PEG, poly(ethylene glycol); PLGA, poly(lactic-co-glycolic acid); SEM, standard error of the mean; TUNEL, terminal deoxynucleotidyl transferase dUTP nick-end labeling.

allowing NPs to evade the immune system. ${ }^{44,46-48}$ Liraglutide can benefit from the advantages offered by the PLGA-PEG delivery system to overcome the short half-life challenges of protein therapy.

As shown in the in vitro studies, the PLGA-PEG NPs were useful in controlling the release behavior of liraglutide for prolonged periods (Figure 2C). In addition, NPs can remain in the myocardium for at least 4 weeks and elicit longterm benefits without inflammation or fibrosis. After 4 weeks, the NPs are gradually degraded into smaller particles, which are susceptible to phagocytosis. ${ }^{42}$ Furthermore, the released liraglutide was effective in activating AKT protein kinase. Thus, the NPs are unaffected by the PLGA-PEG manufacturing procedures and maintained the native properties of liraglutide (Figure 2D, E).

As shown in the in vivo study, the local, controlled, intramyocardial delivery of NP-liraglutide attenuated adverse cardiac remodeling and facilitated angiogenesis after MI. Masson's trichrome staining revealed that an increase in the relative scar thickness and a decrease in the infarct expansion index in the NP-liraglutide group were superior to that in the other three groups (Figure 5). The induction of neovascularization is one of the important elements determining the therapeutic efficacy of treatments for ischemic heart disease, due to its ability to salvage damaged ischemic tissues and initiate self-repair pathways. ${ }^{49}$ Liraglutide has also been 
reported to stimulate neovascularization. ${ }^{37}$ The number of CD31-expressing vessels was significantly increased in the NP-liraglutide group. The NP-liraglutide-induced increases in vascular density would relieve the ischemia in the periinfarct region. Based on these observations, the improved LV function and geometry may be caused by the increase in revascularization. ${ }^{50}$ Furthermore, the NP group exhibited the same vessel densities as the saline-only group, suggesting that NPs did not induce angiogenesis (Figure 6). Furthermore, in this study, the NP-liraglutide treatment suppressed cardiomyocyte apoptosis (Figure 7).

\section{Conclusion}

Based on these results, the NP-liraglutide was useful in controlling the release behavior of liraglutide for prolonged periods. Furthermore, this strategy significantly improved cardiac function following MI by attenuating adverse cardiac remolding, promoting angiogenesis and suppressing apoptosis. Thus, we identified NP-liraglutide as an ideal system for liraglutide delivery into the infarcted myocardium, and it would serve as a potential treatment to prevent adverse cardiac remodeling after MI.

\section{Acknowledgments}

We thank, in particular, Sen li, Mi Zhou, Junfeng Cai, Zhe Wang and Anqing Chen for MI model making. This work was funded by the National Natural Science Foundation of China (Grants 81471779, 31671003, 81671832 and 81571826), Thousand Young Talents Program and the Program for Professor of Special Appointment (Eastern Scholar) at Shanghai Institutions of Higher Learning (No TP2014028).

\section{Disclosure}

The authors report no conflicts of interest in this work.

\section{References}

1. Roger VL, Go AS, Lloyd-Jones DM, et al. Heart disease and stroke statistics - 2011 update: a report from the American Heart Association. Circulation. 2011;123(4):e18-e209.

2. Lloyd-Jones DM, Huffman MD, Karmali KN, et al. Estimating longitudinal risks and benefits from cardiovascular preventive therapies among Medicare patients: the million hearts longitudinal ASCVD risk assessment tool. J Am Coll Cardiol. 2017;69(12):1617-1636.

3. Pascual-Gil S, Garbayo E, Diaz-Herraez P, Prosper F, Blanco-Prieto MJ. Heart regeneration after myocardial infarction using synthetic biomaterials. J Control Release. 2015;203:23-38.

4. Bernstein HS, Srivastava D. Stem cell therapy for cardiac disease. Pediatr Res. 2012;71(4 pt 2):491-499.

5. Segers VF, Lee RT. Protein therapeutics for cardiac regeneration after myocardial infarction. J Cardiovasc Transl Res. 2010;3(5):469-477.

6. Gaffney MM, Hynes SO, Barry F, O'Brien T. Cardiovascular gene therapy: current status and therapeutic potential. Br J Pharmacol. 2007; 152(2):175-188.
7. Action to Control Cardiovascular Risk in Diabetes Study Group, Gerstein HC, Miller ME, et al. Effects of intensive glucose lowering in type 2 diabetes. $N$ Engl J Med. 2008;358(24):2545-2559.

8. Marso SP, Poulter NR, Nissen SE, et al. Design of the liraglutide effect and action in diabetes: evaluation of cardiovascular outcome results (LEADER) trial. Am Heart J. 2013;166(5):823.e5-830.e5.

9. Nikolaidis LA, Mankad S, Sokos GG, et al. Effects of glucagon-like peptide- 1 in patients with acute myocardial infarction and left ventricular dysfunction after successful reperfusion. Circulation. 2004;109(8): 962-965.

10. Noyan-Ashraf MH, Momen MA, Ban K, et al. GLP-1R agonist liraglutide activates cytoprotective pathways and improves outcomes after experimental myocardial infarction in mice. Diabetes. 2009;58(4):975-983.

11. Richards P, Parker HE, Adriaenssens AE, et al. Identification and characterization of GLP-1 receptor-expressing cells using a new transgenic mouse model. Diabetes. 2014;63(4):1224-1233.

12. Inoue $\mathrm{T}$, Inoguchi $\mathrm{T}$, Sonoda $\mathrm{N}$, et al. GLP-1 analog liraglutide protects against cardiac steatosis, oxidative stress and apoptosis in streptozotocininduced diabetic rats. Atherosclerosis. 2015;240(1):250-259.

13. Bugger H, Abel ED. Molecular mechanisms of diabetic cardiomyopathy. Diabetologia. 2014;57(4):660-671.

14. Marre M, Shaw J, Brandle M, et al. Liraglutide, a once-daily human GLP-1 analogue, added to a sulphonylurea over 26 weeks produces greater improvements in glycaemic and weight control compared with adding rosiglitazone or placebo in subjects with Type 2 diabetes (LEAD-1 SU). Diabet Med. 2009;26(3):268-278.

15. Buse JB, Nauck M, Forst T, et al. Exenatide once weekly versus liraglutide once daily in patients with type 2 diabetes (DURATION-6): a randomised, open-label study. Lancet. 2013;381(9861):117-124.

16. Nauck M, Frid A, Hermansen K, et al. Efficacy and safety comparison of liraglutide, glimepiride, and placebo, all in combination with metformin, in type 2 diabetes: the LEAD (liraglutide effect and action in diabetes)-2 study. Diabetes Care. 2009;32(1):84-90.

17. Wu J, Kamaly N, Shi J, et al. Development of multinuclear polymeric nanoparticles as robust protein nanocarriers. Angew Chem Int Ed Engl. 2014;53(34):8975-8979.

18. Xiao Z, Farokhzad OC. Aptamer-functionalized nanoparticles for medical applications: challenges and opportunities. ACS Nano. 2012; 6(5):3670-3676.

19. Xiao Z. CuS nanoparticles: clinically favorable materials for photothermal applications? Nanomedicine. 2014;9(3):373-375.

20. Kamaly N, Xiao Z, Valencia PM, Radovic-Moreno AF, Farokhzad OC. Targeted polymeric therapeutic nanoparticles: design, development and clinical translation. Chem Soc Rev. 2012;41(7):2971-3010.

21. Gu F, Zhang L, Teply BA, et al. Precise engineering of targeted nanoparticles by using self-assembled biointegrated block copolymers. Proc Natl Acad Sci U S A. 2008;105(7):2586-2591.

22. Noyan-Ashraf MH, Shikatani EA, Schuiki I, et al. A glucagon-like peptide-1 analog reverses the molecular pathology and cardiac dysfunction of a mouse model of obesity. Circulation. 2013;127(1):74-85.

23. Ruvinov E, Leor J, Cohen $\mathrm{S}$. The promotion of myocardial repair by the sequential delivery of IGF-1 and HGF from an injectable alginate biomaterial in a model of acute myocardial infarction. Biomaterials. 2011; 32(2):565-578.

24. Ruvinov E, Leor J, Cohen S. The effects of controlled HGF delivery from an affinity-binding alginate biomaterial on angiogenesis and blood perfusion in a hindlimb ischemia model. Biomaterials. 2010;31(16): 4573-4582.

25. Yoshizumi T, Zhu Y, Jiang H, et al. Timing effect of intramyocardial hydrogel injection for positively impacting left ventricular remodeling after myocardial infarction. Biomaterials. 2016;83:182-193.

26. Paul A, Hasan A, Kindi HA, et al. Injectable graphene oxide/hydrogelbased angiogenic gene delivery system for vasculogenesis and cardiac repair. ACS Nano. 2014;8(8):8050-8062.

27. Wang Q, Yang H, Bai A, et al. Functional engineered human cardiac patches prepared from nature's platform improve heart function after acute myocardial infarction. Biomaterials. 2016;105:52-65. 
28. Song M, Jang H, Lee J, et al. Regeneration of chronic myocardial infarction by injectable hydrogels containing stem cell homing factor SDF-1 and angiogenic peptide Ac-SDKP. Biomaterials. 2014;35(8): 2436-2445.

29. Kim JH, Jung Y, Kim SH, et al. The enhancement of mature vessel formation and cardiac function in infarcted hearts using dual growth factor delivery with self-assembling peptides. Biomaterials. 2011; 32(26):6080-6088.

30. Santos-Gallego CG, Vahl TP, Goliasch G, et al. Sphingosine-1-phosphate receptor agonist fingolimod increases myocardial salvage and decreases adverse postinfarction left ventricular remodeling in a porcine model of ischemia/reperfusion. Circulation. 2016;133(10):954-966.

31. Chang MY, Yang YJ, Chang CH, et al. Functionalized nanoparticles provide early cardioprotection after acute myocardial infarction. J Control Release. 2013;170(2):287-294.

32. Sabbah HN, Shimoyama H, Kono T, et al. Effects of long-term monotherapy with enalapril, metoprolol, and digoxin on the progression of left ventricular dysfunction and dilation in dogs with reduced ejection fraction. Circulation. 1994;89(6):2852-2859.

33. Dor V, Sabatier M, Di Donato M, Montiglio F, Toso A, Maioli M. Efficacy of endoventricular patch plasty in large postinfarction akinetic scar and severe left ventricular dysfunction: comparison with a series of large dyskinetic scars. J Thorac Cardiovasc Surg. 1998;116(1):50-59.

34. D'Amore A, Yoshizumi T, Luketich SK, et al. Bi-layered polyurethaneextracellular matrix cardiac patch improves ischemic ventricular wall remodeling in a rat model. Biomaterials. 2016;107:1-14.

35. Marso SP, Daniels GH, Brown-Frandsen K, et al. Liraglutide and cardiovascular outcomes in type 2 diabetes. $N$ Engl J Med. 2016; 375(4):311-322.

36. Kavianipour M, Ehlers MR, Malmberg K, et al. Glucagon-like peptide-1 (7-36) amide prevents the accumulation of pyruvate and lactate in the ischemic and non-ischemic porcine myocardium. Peptides. 2003; 24(4):569-578.

37. Sato K, Kameda M, Yasuhara T, et al. Neuroprotective effects of liraglutide for stroke model of rats. Int J Mol Sci. 2013;14(11):21513-21524.

38. Lee J, Tan CY, Lee SK, Kim YH, Lee KY. Controlled delivery of heat shock protein using an injectable microsphere/hydrogel combination system for the treatment of myocardial infarction. J Control Release. 2009;137(3):196-202.

39. Wang L, Liu Y, Zhang W, Chen X, Yang T, Ma G. Microspheres and microcapsules for protein delivery: strategies of drug activity retention. Curr Pharm Des. 2013;19(35):6340-6352.
40. Wu J, Williams GR, Branford-White C, Li H, Li Y, Zhu LM. Liraglutide-loaded poly(lactic-co-glycolic acid) microspheres: preparation and in vivo evaluation. Eur J Pharm Sci. 2016;92:28-38.

41. Formiga FR, Pelacho B, Garbayo E, et al. Sustained release of VEGF through PLGA microparticles improves vasculogenesis and tissue remodeling in an acute myocardial ischemia-reperfusion model. J Control Release. 2010;147(1):30-37.

42. Pascual-Gil S, Simon-Yarza T, Garbayo E, Prosper F, Blanco-Prieto MJ. Tracking the in vivo release of bioactive NRG from PLGA and PEGPLGA microparticles in infarcted hearts. J Control Release. 2015; 220(pt A):388-396.

43. Qiao S, Zhao Y, Geng S, et al. A novel double-targeted nondrug delivery system for targeting cancer stem cells. Int J Nanomedicine. 2016; 11:6667-6678.

44. $\mathrm{Bi} \mathrm{C}$, Wang $\mathrm{A}, \mathrm{Chu} \mathrm{Y}$, et al. Intranasal delivery of rotigotine to the brain with lactoferrin-modified PEG-PLGA nanoparticles for Parkinson's disease treatment. Int J Nanomedicine. 2016;11:6547-6559.

45. Jahan ST, Haddadi A. Investigation and optimization of formulation parameters on preparation of targeted anti-CD205 tailored PLGA nanoparticles. Int J Nanomedicine. 2015;10:7371-7384.

46. Simon-Yarza T, Formiga FR, Tamayo E, Pelacho B, Prosper F, BlancoPrieto MJ. PEGylated-PLGA microparticles containing VEGF for long term drug delivery. Int J Pharm. 2013;440(1):13-18.

47. Gref R, Luck M, Quellec P, et al. 'Stealth' corona-core nanoparticles surface modified by polyethylene glycol (PEG): influences of the corona (PEG chain length and surface density) and of the core composition on phagocytic uptake and plasma protein adsorption. Colloids Surf B Biointerfaces. 2000;18(3-4):301-313.

48. Wattendorf U, Merkle HP. PEGylation as a tool for the biomedical engineering of surface modified microparticles. J Pharm Sci. 2008; 97(11):4655-4669.

49. Haider H, Akbar SA, Ashraf M. Angiomyogenesis for myocardial repair. Antioxid Redox Signal. 2009;11(8):1929-1944.

50. Gyongyosi M, Khorsand A, Zamini S, et al. NOGA-guided analysis of regional myocardial perfusion abnormalities treated with intramyocardial injections of plasmid encoding vascular endothelial growth factor A-165 in patients with chronic myocardial ischemia - subanalysis of the EUROINJECT-ONE multicenter double-blind randomized study. Circulation. 2005;112(9):I157-I165. 


\section{Supplementary materials}

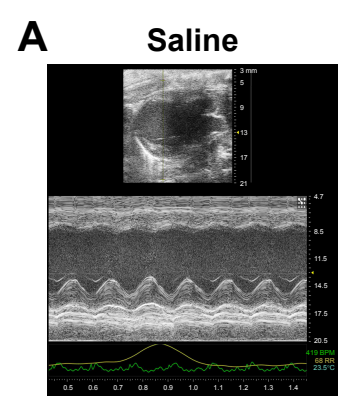

B

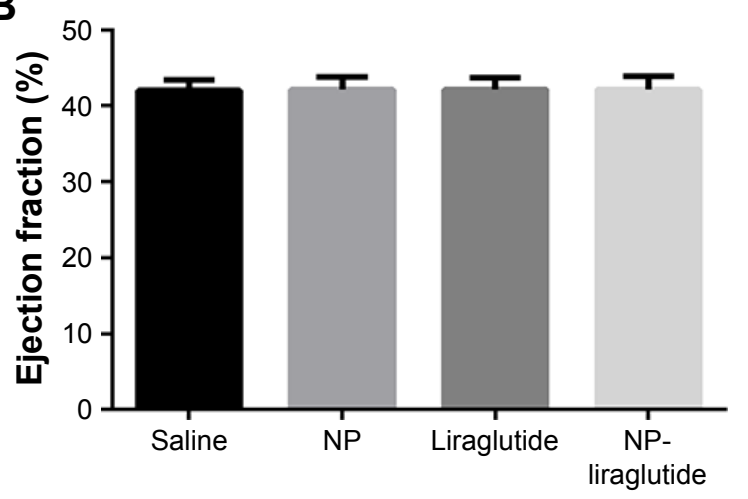

D

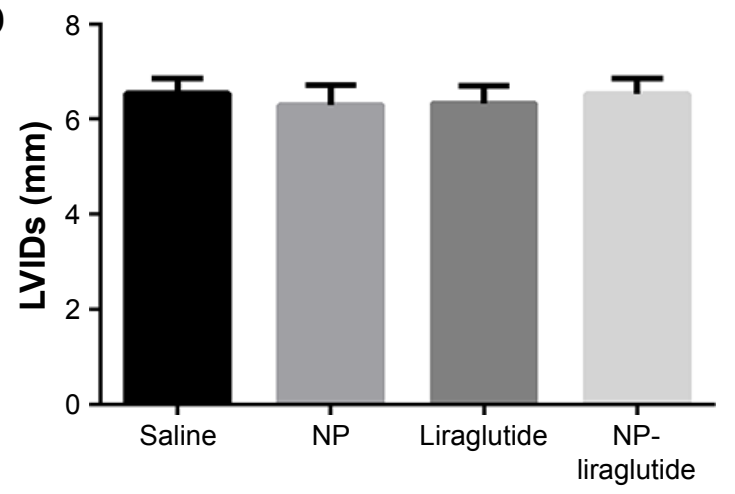

C

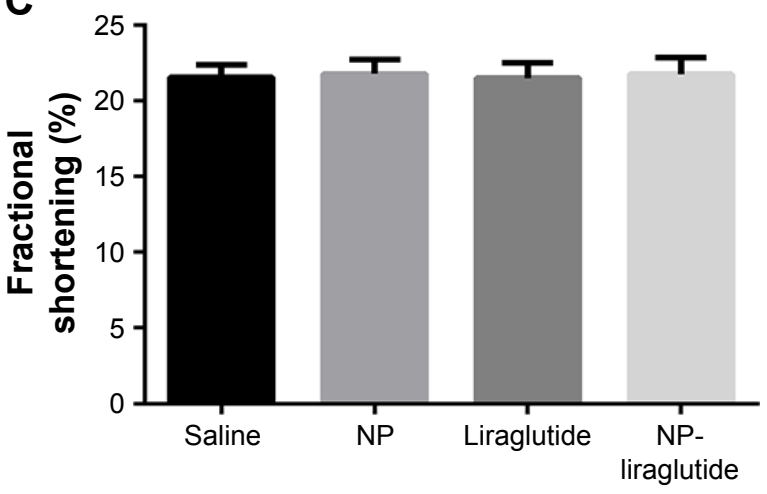

E

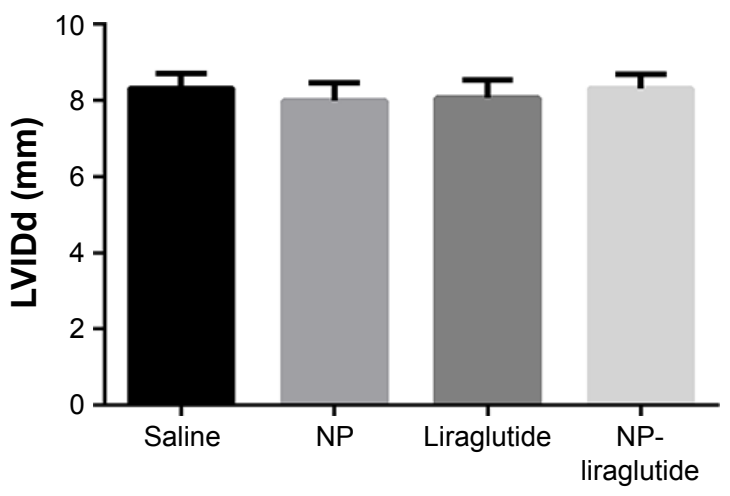

Saline

NP

Liraglutide

NP-liraglutide

Figure SI Echocardiographic assessments.

Notes: (A) Representative echocardiographic images from each group 2 days after ligation. The LVEF (B), LVFS (C), LVIDs (D) and LVIDd (E) were assessed via twodimensional echocardiography. The change in each measurement is summarized. Data are shown as mean \pm SEM. NP-liraglutide: liraglutide loaded in PLGA-PEG NPs. Abbreviations: LVEF, left ventricular ejection fraction; LVFS, left ventricular fractional shortening; LVIDs, left ventricular internal systolic dimension; LVIDd, left ventricular internal diastolic dimension; NP, nanoparticle; PEG, poly(ethylene glycol); PLGA, poly(lactic-co-glycolic acid); SEM, standard error of the mean. 


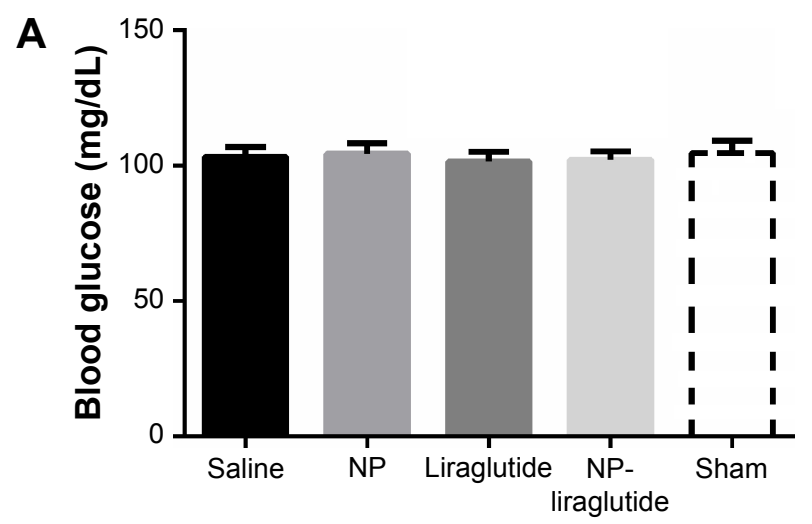

1 week

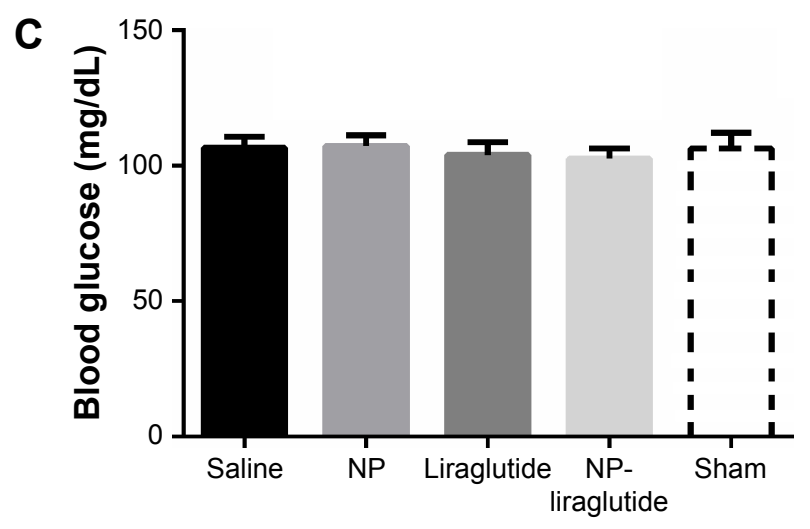

3 weeks

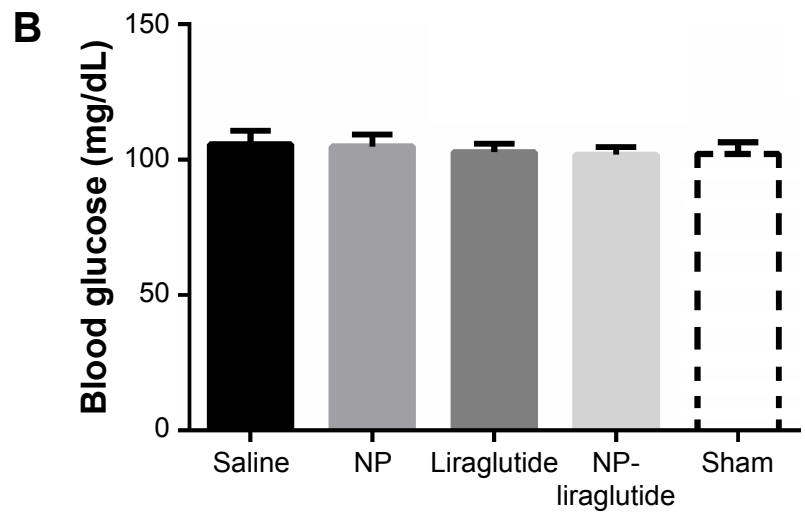

2 weeks

D

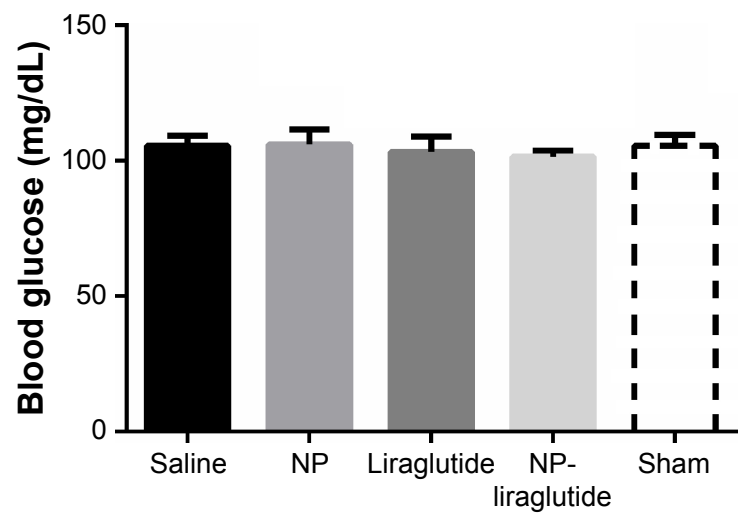

4 weeks

Figure S2 Blood glucose levels.

Notes: The blood glucose was measured at I week (A), 2 weeks (B), 3 weeks (C) and 4 weeks (D). NP-liraglutide had no significant effects on random blood glucose levels among different groups. Data shown as mean \pm SEM.

Abbreviations: NP, nanoparticle; SEM, standard error of the mean.

\section{Publish your work in this journal}

The International Journal of Nanomedicine is an international, peerreviewed journal focusing on the application of nanotechnology in diagnostics, therapeutics, and drug delivery systems throughout the biomedical field. This journal is indexed on PubMed Central, MedLine, CAS, SciSearch $₫$, Current Contents $₫ /$ Clinical Medicine,
Journal Citation Reports/Science Edition, EMBase, Scopus and the Elsevier Bibliographic databases. The manuscript management system is completely online and includes a very quick and fair peer-review system, which is all easy to use. Visit http://www.dovepress.com/ testimonials.php to read real quotes from published authors. 